\title{
LA ENTONACIÓN DE LOS ENUNCIADOS DECLARATIVOS DE FOCO AMPLIO EN EL HABLA LIMEÑA
}

\section{INTONATION OF BROAD FOCUS DECLARATIVE STATEMENTS IN LIMA'S SPEECH}

\author{
Olga Lucía Garzón Acuña \\ Universidad Autónoma de Querétaro \\ garzonolgalu@gmail.com \\ https://orcid.org/0000-0002-4130-9886 \\ Eva Patricia Velásquez Upegui \\ Universidad Autónoma de Querétaro \\ evapvelasquez@gmail.com \\ https://orcid.org/0000-0001-6779-7331
}

Recibido: $31 / 07 / 2019$

Aceptado: 28/04/2019

\section{Resumen}

En esta investigación se observan las características entonativas de los enunciados declarativos de foco amplio en el habla limeña, como parte del proyecto de Prosodia Basada en el Uso (PBU) en los corpus PRESEEA (Martín Butragueño y Velásquez-Upegui, 2014). El estudio se fundamenta en los principios del Modelo Métrico Autosegmental (AM). De acuerdo con la metodología propuesta en PBU, se consideran las variaciones en la frecuencia $\left(f_{0}\right)$ de las sílabas del tonema. Así mismo, se estudia la declinación tonal entre el tono básico y el tono final de los enunciados. En la descripción de los datos se analiza el efecto de las variables sexo, edad

\begin{abstract}
In this research, the intonation features of broad focus declarative statements in Lima's speech are observed as part of the Use-Based Prosody (PBU by its Spanish acronym) project in the PRESEEA corpora (Martín Butragueño and Velásquez-Upegui, 2014). The study is based on the principles of the Autosegmental Metric Model (AM). According to the methodology proposed by the PBU, acoustic variations in the frequency $\left(f_{0}\right)$ of the toneme syllables (nuclear syllable plus juncture material) are considered. Likewise, the tonal decline between the base and final tone of the statements is studied. In the data description, the impact of the sex, age and
\end{abstract}

Para citar este artículo / To cite this article: Garzón Acuña, Olga Lucía y Velásquez Upegui, Eva Patricia (2020). La entonación de los enunciados declarativos de foco amplio en el habla limeña. ELUA, 34: 117-129. https://doi.org/10.14198/ELUA2020.34.5

Enlace / Link: https://doi.org/10.14198/ELUA2020.34.5 
y grado de instrucción, en el movimiento de la $f_{0}$ en los contextos mencionados. Los resultados indican que las variables de estratificación social inciden en las variaciones de la $f_{0}$ entre las sílabas prenuclear y nuclear, concretamente, las variables de sexo y edad.

PALABRAS CLAVE: Entonación, habla, uso, variación, estratificación. level of education variables on the movement of the $f_{0}$ in the referred contexts is analyzed. The results show that the social stratification variables have a direct impact on the $f_{0}$ variations between the pre-nuclear and nuclear syllables, namely, the sex and age variables.

KEYWORDS: Intonation, speech, use, variation, stratification.

\section{INTRODUCCIÓN}

El estudio de la prosodia de una lengua supone diversos retos metodológicos y descriptivos. Uno de ellos tiene que ver con el reconocimiento de las características entonacionales del habla en uso, es decir, en contextos naturales en los que surgen los intercambios comunicativos. Se hace necesario dar cuenta de las dinámicas sociales que involucran el entorno lingüístico, a partir de las características sociales y culturales de los interlocutores, es decir, del estudio de la prosodia basada en el uso (PBU) (Martín Butragueño y Velásquez-Upegui, 2014). Por lo tanto, un análisis prosódico debe enfocarse en determinar cómo las variables intra y extralingüísticas modifican la realización prosódica de los enunciados.

En este sentido, el proyecto PBU pretende "describir y analizar los patrones prosódicos del español desde un enfoque realista que contribuyan al reconocimiento de procesos de cambio y variación lingüística, social y regional" (Velásquez-Upegui y Martín Butragueño, 2018:1) dentro del corpus PRESEEA. Para cumplir con este objetivo la primera tarea es construir un panorama general de la entonación a partir de la descripción de cada una de las variedades que conforman este corpus. En principio, se toman como objeto descriptivo los enunciados declarativos de foco amplio, partiendo de la hipótesis de la existencia de una entonación de base (no marcada) que permita la comparación intradialectal e interdialectal que permita trazar convergencias y divergencias en el habla hispana.

De acuerdo con Martín-Butragueño y Velásquez-Upegui (2014), esta primera descripción aborda la entonación desde una perspectiva lingüística, es decir, desde la "función distintiva entre los tipos de enunciados"; una perspectiva comunicativa entendida como variación "en función de la estratificación social y la distribución regional de las variedades lingüísticas"; desde una perspectiva pragmática en tanto "constituye un indicador de la fuerza ilocutiva"; y, por último, desde un enfoque discursivo, dado que "constituye una estrategia dialógica con fines interaccionales".

En esta investigación, se plantea como objetivo principal el identificar aspectos prosódicos del habla limeña como parte del panorama entonacional panhispánico en el corpus PRESEEA $^{1}$, se busca describir características de las realizaciones entonativas en enunciados declarativos de foco amplio a través del análisis del tonema, es decir, teniendo en cuenta patrones a nivel local del enunciado que permitan reconocer, en principio, si estas unidades pueden modificarse en función de las características sociales que identifican a los hablantes. En adelante se presentan brevemente los antecedentes sobre la prosodia en

1 Las autoras agradecen muy especialmente a la Dra. Rocío Caravedo, coordinadora del Corpus sociolingüístico del español de Lima, por el apoyo con el envío de los materiales completos de los 18 informantes analizados. 
el habla limeña; a continuación se explica la metodología, seguida por los resultados de esta primera aproximación para terminar con las conclusiones del estudio.

\section{ALGUNAS CONSIDERACIONES SOBRE EL ESPAÑOL HABLADO EN LIMA}

La entonación de enunciados declarativos en español se ha caracterizado por presentar un final típicamente descendente en el tonema, es decir, desde la sílaba nuclear a la juntura terminal (Navarro Tomás, 1948, Quilis, 1993). Sosa (1999:140) asocia esta declinación regular en español con el tono L* L\%. Diferentes estudios geoprosódicos desarrollados en la actualidad han indicado que este tipo de realización entonativa se modifica según la variedad dialectal (Prieto y Roseano, 2010; Hualde y Prieto, 2015), sin embargo, las variaciones encontradas parecen alojarse en la sílaba nuclear y muestran coincidencia en el tono descendente de la juntura terminal.

A propósito del español limeño, O’Rourke (2005) estudia las características entonativas de los enunciados declarativos de foco amplio y foco estrecho en el español de Lima y Cuzco en contacto con el Quechua. La autora emplea el Modelo Métrico Autosegmental para analizar los contornos entonativos en los enunciados desde una perspectiva sociolingüística. Los participantes son nativo hablantes de español, nativo hablantes bilingües de español y Quechua y nativo hablantes de Quechua, quienes se encuentran aprendiendo español como L2.

En el caso del habla limeña, la autora determina que el alineamiento de la $f_{0}$ en los acentos prenucleares, se alcanza en la sílaba postónica en los enunciados declarativos de foco amplio. O'Rourke propone que estos enunciados presentan acentos tonales $\mathrm{L}^{*} \mathrm{H}$ y $\mathrm{L}^{*} \mathrm{H}^{*}$ en las sílabas prenucleares, y una configuración nuclear $\mathrm{L}^{*}$ y $\mathrm{H}^{*}$.

García (2011) analiza los patrones entonativos del español amazónico peruano comparando el habla de Lima y Pucallpa. En su estudio observa las características de los enunciados declarativos de foco amplio, foco estrecho y foco contrastivo. En cuanto al alineamiento, se encuentra que, en los acentos prenucleares, el pico tonal se desplaza hasta la sílaba postónica (O’Rourke, 2005), y es representado como L $+\mathrm{H}^{*}$.

Con respecto a la duración vocálica en el español de Lima, García (2014) realiza un estudio experimental exploratorio en el español amazónico peruano, contrastando los hallazgos con la variedad limeña (grupo control). La autora elaboró un corpus de 10 oraciones declarativas de foco amplio con el orden SVO y tres palabras léxicas, todas trisilábicas y llanas. Las variables lingüísticas consideradas fueron el acento (vocales tónicas y átonas) y su posición en la oración (final y no final).

La autora centra su atención en las vocales tónicas y pretónicas, localizadas en posición del verbo y del objeto. En el análisis fueron incluidas solamente aquellas ubicadas dentro de sílabas abiertas, evitando así posibles efectos de la estructura silábica sobre la duración vocálica. Los resultados indican que, en la variedad amazónica, las vocales son fonéticamente más largas que aquellas de la variedad limeña, y que dicha diferencia se hace más evidente en las vocales de las sílabas tónicas en posición final. Con relación al desplazamiento del pico tonal, García (2014) apunta que se presenta hasta después de la sílaba tónica, particularmente en los acentos prenucleares de las 'oraciones declarativas neutras'. En posición nuclear, en cambio, este pico se realiza dentro de la sílaba tónica.

García (2016) busca examinar la alineación tonal de acentos crecientes en el español amazónico peruano, en la ciudad de Pucallpa. Se recolectaron datos de 13 hablantes mono- 
lingües por medio de una tarea de lectura y una entrevista. En la tarea de lectura, se seleccionaron enunciados de foco amplio, estrecho y contrastivo. En la entrevista, se pidió a los oradores que hablaran sobre un tema en particular durante uno o dos minutos.

El análisis se centró en tres variables dependientes: la ubicación del pico tonal, el tiempo de subida de la $f_{0}$ y la altura alcanzada. Las variables independientes fueron: número de palabras, estructura de sílabas, posición de la palabra y tipo de foco. A partir de la observación de todos estos aspectos, García (2016) propone una configuración nuclear L+H* para los enunciados declarativos de foco amplio.

\section{METODOLOGÍA}

\subsection{Participantes}

De acuerdo con las consideraciones metodológicas del proyecto PBU (VelásquezUpegui y Martín-Butragueño, 2018), se ha determinado una muestra conformada por 18 informantes (1 por cada casilla), Se establece un diseño de 18 hablantes x 2 sexos x 3 edades x 3 niveles de instrucción. Es decir, un hombre y una mujer por cada generación; generación 1, de 20 a 34 años; generación 2, de 35 a 54 años; generación 3, de 55 años en adelante y, de igual manera, por cada grado de instrucción; grado 1, personas analfabetas, sin estudios, enseñanza primaria (hasta 10-11 años de edad aprox.), cinco años aproximadamente de escolarización; grado 2, enseñanza secundaria (hasta 16-18 años de edad aprox.), 10-12 años aproximadamente de escolarización; grado 3, enseñanza superior (universitaria, técnica superior) (hasta 21-22 años de edad aprox.), 15 años aproximadamente de escolarización.

\subsection{Procedimiento}

Se parte del enfoque del Modelo Métrico Autosegmental (AM) (Prieto, 2003; Hualde, 2003, Pierrehumbert, 1980; Pierrehumbert y Beckman, 1988) que está diseñado para dar cuenta de los elementos contrastivos del sistema entonativo. Este abordaje permite la descripción de los contornos melódicos presentes en los enunciados objeto de estudio. Adicionalmente, el modelo permite identificar los segmentos fonológicos que componen los enunciados, los cuales se etiquetan con el sistema de notación $S p \_$ToBI (Tones and Break Indices) (Estebas y Prieto, 2008).

\subsubsection{Selección de enunciados}

La selección de tokens se realiza considerando cinco enunciados declarativos de foco amplio por cada participante, todos los enunciados que se extrajeron de las entrevistas sociolingüísticas corresponden a actos de habla asertivos (Searle, 2010) o representativos (Yule, 1996). Este tipo de actos de habla se asocia prosódicamente con una entonación no marcada o neutra, es decir, constituye un foco amplio o también llamado foco presentativo o informativo, en el que el foco o realce coincide con toda la información contenida en el enunciado. Los focos amplios, por lo regular, proporcionan respuestas a preguntas en las que no se identifica o destaca algún elemento en particular (Gutiérrez Bravo, 2008). 
Los enunciados que conforman el corpus final de estudio corresponden a frases fonológicas localizadas en el límite derecho, atendiendo al concepto de acto y subacto de habla (Hidalgo y Padilla, 2006), con el tonema alojado en palabra llana. El total de enunciados estudiados fue de 90 (45 de hombres y 45 de mujeres), seleccionados del corpus PRESEEALima (Caravedo, 2008).

\subsubsection{Tratamiento de los datos}

El análisis acústico se llevó a cabo con ayuda del software Praat, para realizar la medición de los puntos de inicio y final de los enunciados, y las sílabas que conforman el tonema, considerando la sílaba prenuclear, inclusive. Inicialmente, se restauró el pitch en los enunciados donde la lectura de la $f_{0}$ se encontraba alterada por el ruido, por el efecto de voz laringizada en el contorno melódico o por una caída del tono generada por efectos microprosódicos.

Se calculó el promedio de la $f_{0}$ de las vocales en cada sílaba del tonema, y se establecieron las mediciones en semitonos (st.). Se calculó la diferencia entre los valores obtenidos en las sílabas del tonema. Asimismo, se establecieron los promedios de inicio y final de los enunciados. Se calculó el descenso de la $f_{0}$ en la sílaba postnuclear con respecto al tono básico ${ }^{2}$ de los informantes (Ávila, 2003) y, por último, se construyó la base de datos con los valores normalizados y las transcripciones fonéticas.

El etiquetado de los enunciados se efectuó teniendo en cuenta la representación tonal en cada enunciado a partir del movimiento de la curva tonal y de la diferencia alcanzada en semitonos (st). de acuerdo con el criterio del umbral de percepción de 1.5 st (Pamies, Fernández, Martínez, Ortega y Amorós, 2002). Los movimientos menores a 1.5 st, se transcribieron con monotonos $\left(\mathrm{H}^{*}, \mathrm{~L}^{*}\right)$ y para los movimientos mayores a 1.5 st se reservaron los bitonos $\left(\mathrm{L}+\mathrm{H}^{*}, \mathrm{~L}^{*}+\mathrm{H}, \mathrm{L}+>\mathrm{H}^{*}, \mathrm{H}+\mathrm{L}^{*}\right)$. Para los tonos de juntura terminal se emplearon los monotonos L\%, M\% y H\%. (Estebas-Vilaplana y Prieto, 2008).

Para el análisis estadístico se empleó el paquete SPSS (Statistical Package for Social Sciences). Se estableció como variable dependiente la medida de la $f_{0}$ en las sílabas analizadas. Se llevó a cabo una ANOVA de un factor y se aplicó el ajuste post hoc de Bonferroni. En los casos en que no se cumple el supuesto de normalidad se realizó la prueba no paramétrica de Kruskal Wallis.

\section{RESULTADOS}

El análisis de los datos permite observar que la configuración nuclear más frecuente corresponde a un tono sostenido $\mathrm{H}^{*}$. Esta representación entonacional en los enunciados declarativos de foco amplio, corresponde a un acento nuclear monotonal que describe una trayectoria plana o con inflexiones menores a 1.5 st. que se mantienen, incluso, hasta la juntura terminal M\% (Figura 1). El segmento prenuclear presenta un ascenso en la sílaba tónica anterior al núcleo $\mathrm{L}+\mathrm{H}^{*}$, seguido de un descenso $\mathrm{y}$, posterior, sostenimiento al final del enunciado o, en algunos casos, la finalización con un descenso continuo. En el ejemplo se observa estabilidad en las flexiones de la $f_{0}$ en el tonema formado por la última sílaba

2 Promedio tonal de todas las mediciones de la $f_{0}$ en la vocal al inicio de los enunciados, por cada participante. 
léxicamente acentuada - $m a$-, a la que está anclado el acento nuclear $\mathrm{H}^{*} \mathrm{y}$, por la sílaba postónica -no-, cuyo linde está alineado con el tono de juntura final $\mathrm{M} \%$.

LIMA HII US

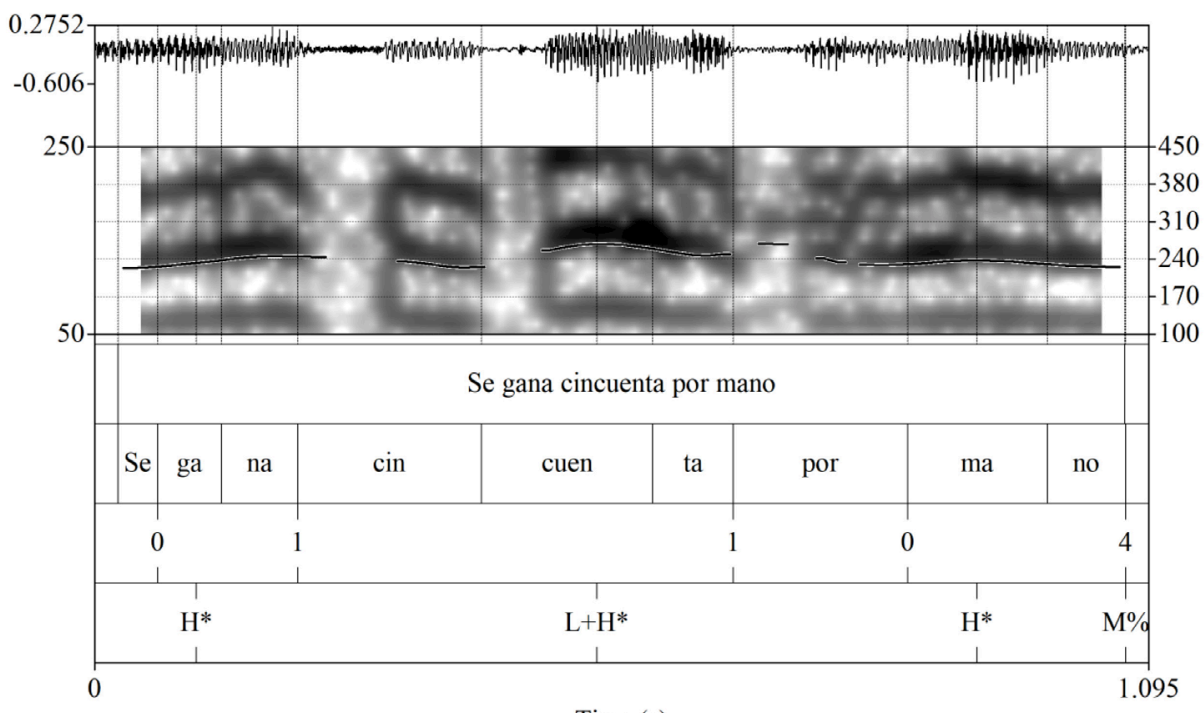

Time (s)

Figura 1. Enunciado declarativo de foco amplio. Hombre, generación 1, nivel de instrucción 1 [...] Se gana cincuenta por mano

Aunque la representación más frecuente en el tono nuclear corresponde a $\mathrm{H}^{*}$, es posible encontrar otras realizaciones de menor frecuencia (Tabla 1). Se trata de un movimiento ascendente $\mathrm{L}+\mathrm{H}^{*}$ y otro descendente $\mathrm{H}+\mathrm{L}^{*}$. Así mismo, se hallan tres tonos de juntura terminal; sostenido $\mathrm{M} \%$, descendente $\mathrm{L} \%$ y ascendente $\mathrm{H} \%$, de mayor a menor frecuencia, respectivamente. De acuerdo con lo anterior, se podría plantear la existencia de dos configuraciones más $\mathrm{L}+\mathrm{H}^{*} \mathrm{~L} \% \mathrm{y}$, por último, la configuración $\mathrm{H}+\mathrm{L} * \mathrm{~L} \%$, aunque no son los más recurrentes.

\begin{tabular}{|c|c|c|c|c|c|}
\hline \multirow{2}{*}{\multicolumn{2}{|c|}{ TONEMA }} & \multicolumn{3}{|c|}{ JUNTURA TERMINAL } & \multirow{3}{*}{$\begin{array}{l}\text { Total tonos nucleares } \\
57 \\
(63.33 \%)\end{array}$} \\
\hline & & \multirow{2}{*}{$\begin{array}{c}\mathrm{H} \% \\
2 \\
(2.22 \%)\end{array}$} & \multirow{2}{*}{$\begin{array}{c}\mathrm{M} \% \\
34 \\
(37.77 \%)\end{array}$} & \multirow{2}{*}{$\begin{array}{c}\mathrm{L} \% \\
21 \\
(23.33 \%)\end{array}$} & \\
\hline \multirow{3}{*}{$\begin{array}{c}\text { SÍLABA } \\
\text { NUCLEAR }\end{array}$} & $\mathrm{H}^{*}$ & & & & \\
\hline & $\mathrm{L}+\mathrm{H}^{*}$ & $\begin{array}{c}0 \\
(0 \%)\end{array}$ & $\begin{array}{c}4 \\
(4.44 \%)\end{array}$ & $\begin{array}{c}14 \\
(15.55 \%)\end{array}$ & $\begin{array}{c}18 \\
(20 \%)\end{array}$ \\
\hline & $\mathrm{H}+\mathrm{L}^{*}$ & $\begin{array}{c}0 \\
(0 \%)\end{array}$ & $\begin{array}{c}6 \\
(6.66 \%)\end{array}$ & $\begin{array}{c}9 \\
(10 \%)\end{array}$ & $\begin{array}{c}15 \\
(16.66 \%)\end{array}$ \\
\hline \multicolumn{2}{|c|}{$\begin{array}{c}\text { Total tonos de juntura } \\
\text { Terminal }\end{array}$} & $\begin{array}{c}2 \\
(2.22 \%)\end{array}$ & $\begin{array}{c}44 \\
(48.88 \%)\end{array}$ & $\begin{array}{c}44 \\
(48.88 \%)\end{array}$ & $\begin{array}{c}90 \\
(100 \%)\end{array}$ \\
\hline
\end{tabular}

Tabla 1. Frecuencia de acentos nucleares y junturas terminales en el habla limeña 
Con respecto a los movimientos de la $f_{0}$ en el tonema, se encuentra una diferencia promedio de 0.03 st. \pm 2.51 entre las sílabas prenuclear y nuclear; y un descenso de 2.04 st. \pm 3.52 entre la sílaba nuclear y postnuclear (Figura 2). Es decir, una realización plana en la sílaba tónica, seguida por un descenso.

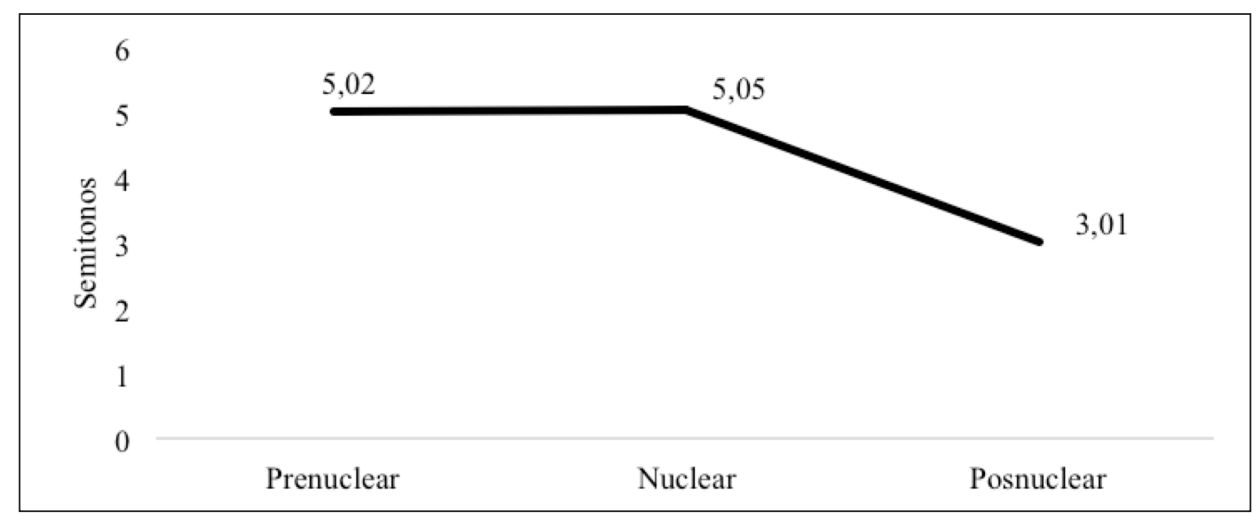

Figura 2. Media de las diferencias de $f_{0}$ en el tonema

\subsection{La $f_{0}$ y las variables sexo, edad y nivel de instrucción}

Con relación a la variable sexo, se encuentra que influye significativamente en el movimiento de la $f_{0}$ en las sílabas prenuclear y nuclear, $\mathrm{X}^{2}$ (1) $6.278 \mathrm{p}<0.05$. En el caso de las mujeres presentan un movimiento descendente de $0.62 \mathrm{st} \pm 2.64$, en comparación con los hombres quienes presentaron un incremento de $0.67 \mathrm{st} . \pm 2.22$ en la $f_{0}$ entre estas sílabas (Figura 3).

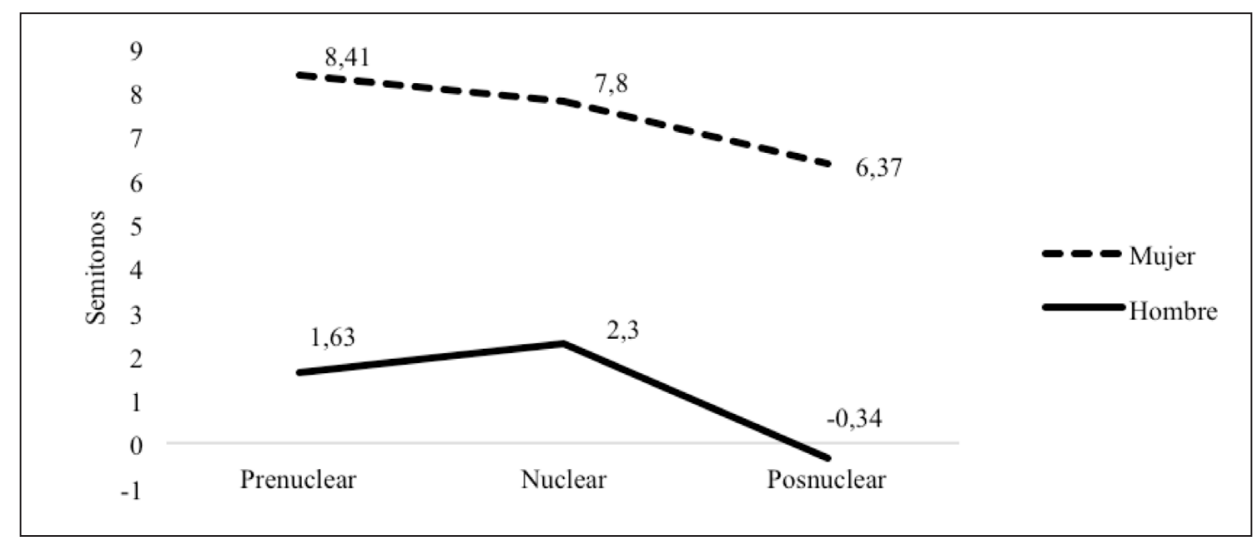

Figura 3. Medias de la $f_{0}$ entre las sílabas del tonema por variable sexo 
En el trayecto de la sílaba nuclear y posnuclear ${ }^{3}$ no se presentó un efecto significativo del sexo en la $f_{0}, \mathrm{~F}(1,72)=2.856, \mathrm{p}>0.05$. En ambos grupos se encontró un movimiento descendente que, solo en el caso de los hombres, excede el umbral perceptivo de 1.5 st (Tabla 2).

\begin{tabular}{ccccccc}
\hline \multirow{2}{*}{ Variable } & \multicolumn{2}{c}{ Sílaba Prenuclear-Nuclear } & \multicolumn{3}{c}{ Sílaba Nuclear-Posnuclear } & \\
& & Media & Desv.Est & Media & Desv.Est & Total \\
\hline \multirow{2}{*}{ Sexo } & Mujeres & -0.61 & 2.64 & -1.43 & 3.37 & 45 \\
& Hombres & 0.67 & 2.22 & -2.64 & 3.6 & 45 \\
\hline
\end{tabular}

Tabla 2. Medias de la $f_{0}$ entre las sílabas del tonema por variable sexo ${ }^{4}$

$\mathrm{Al}$ observar las realizaciones entonativas por grupos etarios, se encuentra que esta variable de estratificación afecta la altura alcanzada en el tonema. La edad tiene un efecto significativo en el movimiento de la $f_{0}$ entre las sílabas prenuclear y nuclear $\mathrm{X}^{2}(2) 6.814 \mathrm{p}<0.05$. Las comparaciones múltiples en este segmento indican que la diferencia es significativa entre las generaciones 2 y 3 , exclusivamente $\mathrm{p}<0.05$. La generación 2 revela un movimiento descendente $(0.55 \mathrm{st})$, mientras que la generación 3 presenta un ascenso de la $f_{0}(1.04 \mathrm{st})$. En cuanto a la $f_{0}$ entre la sílaba nuclear y posnuclear, el análisis estadístico reveló que la edad no tiene un efecto significativo en su altura $F(2,72)=2.969, \mathrm{p}>0.05$.

\begin{tabular}{lcccccc}
\hline \multirow{2}{*}{ Variable } & \multicolumn{3}{c}{ Sílaba Prenuclear-Nuclear } & \multicolumn{2}{c}{ Sílaba Nuclear- Posnuclear } & \\
& & Media & Desv.Est & Media & Desv.Est & Total \\
\hline \multirow{3}{*}{ Generación } & G1 & -0.38 & 2.68 & -1.19 & 3.62 & 30 \\
& G2 & -0.55 & 2.5 & -1.68 & 3.03 & 30 \\
& G3 & 1.04 & 2.07 & -3.24 & 3.67 & 30 \\
\hline
\end{tabular}

Tabla 3. Medias de la $f_{0}$ entre las sílabas del tonema por variable generación

Los hablantes de la generación 3 (mayores de 55 años) presentaron un movimiento ascendente en la sílaba nuclear, aspecto que no evidenciaron los demás grupos generacionales. Los hablantes de los grupos 1 y 2, por su parte, realizaron movimientos descendentes. Se observa un descenso menor en el caso de los hablantes del grupo 1 (20 a 35 años) con respecto los hablantes del grupo 2 (35 y 54 años) (Figura 4). En los tres grupos de hablantes el movimiento hacia el final del tonema es descendente. En esta realización, la generación 3 se distingue por una declinación mayor que excede el umbral de percepción (1.5 st) y constituye casi el doble del declive presentado en los grupos de las generaciones 1 y 2.

3 No se encontró un efecto estadísticamente significativo entre las variables sexo, edad y grado de instrucción en la altura de $f_{0}$ en el trayecto de la sílaba nuclear a la posnuclear $\mathrm{F}(17,72)=1.412$, $\mathrm{p}>0.05$.

4 El signo negativo en las tablas se emplea exclusivamente para indicar la dirección descendente del movimiento de la $f_{0}$. 


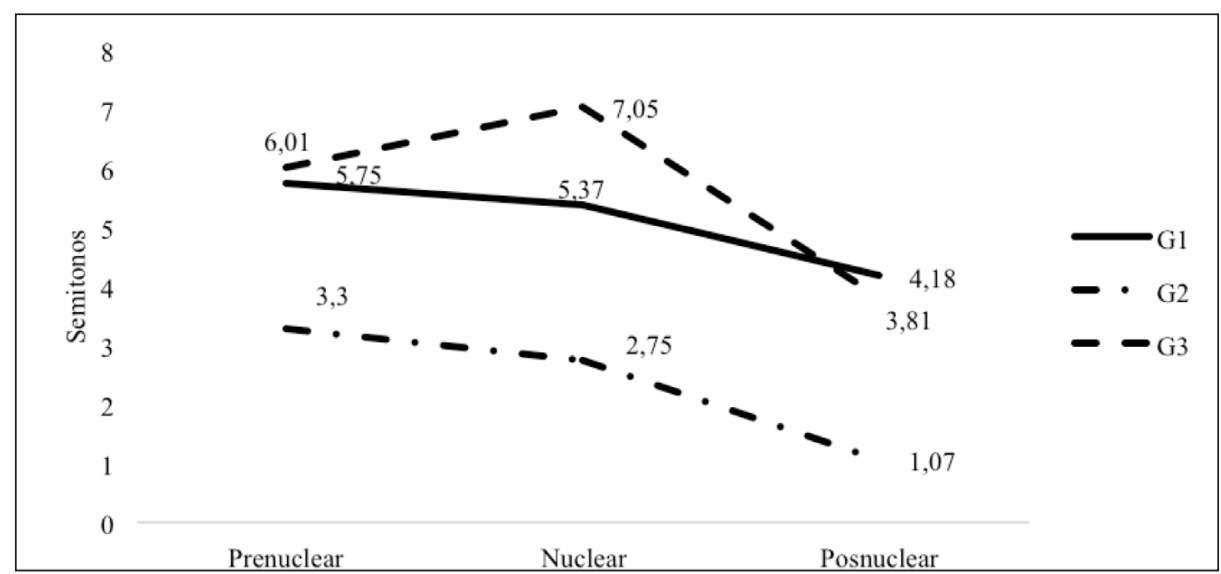

Figura 4. Medias de la $f_{0}$ entre las sílabas del tonema por variable generación

Con respecto al nivel de instrucción, no se encuentran diferencias estadísticamente significativas para ninguno de los grupos. Es decir, la variable nivel de instrucción no influye significativamente en el movimiento de la $f_{0}$ en la sílaba nuclear $\mathrm{X}^{2}$ (2) $3.359 \mathrm{p}>0.05$ ni en la juntura terminal $\mathrm{F}(2,72)=1.123, \mathrm{p}>0.05$. (Tabla 4). Las trayectorias tonales que presenta cada nivel de instrucción se observan en la figura 5.

\begin{tabular}{lcccccc}
\hline \multirow{2}{*}{ Variable } & \multicolumn{3}{c}{ Sílaba Prenuclear Nuclear } & \multicolumn{4}{c}{ Sílaba Nuclear-Posnuclear } \\
& & Media & Desv.Est & Media & Desv.Est & Total \\
\hline \multirow{3}{*}{ Grado de Instruc. } & G1 & -0.11 & 2.36 & -2.38 & 2.9 & 30 \\
& G2 & -0.53 & 2.72 & -1.28 & 4.02 & 30 \\
& G3 & 0.75 & 2.34 & -2.34 & 3.56 & 30 \\
\hline
\end{tabular}

Tabla 4. Medias de la $f_{0}$ entre las sílabas del tonema por variable grado de instrucción

Se observa que las realizaciones entonativas presentan diferencias para cada grado de instrucción, a pesar de no tener un efecto estadísticamente significativo. Los hablantes con menor grado de instrucción (Grado 1) mostraron un movimiento sostenido en la sílaba nuclear a diferencia de los demás grupos. Los hablantes del Grado 2 mantuvieron un ligero descenso, mientras que los hablantes con el grado más alto de instrucción (Grado 3 ) presentaron un movimiento ascendente (Figura 5). En los tres grados de instrucción se presentan finales descendentes de diferente magnitud, sólo en los grupos 1 y 3 se excede el umbral de 1.5 st. 


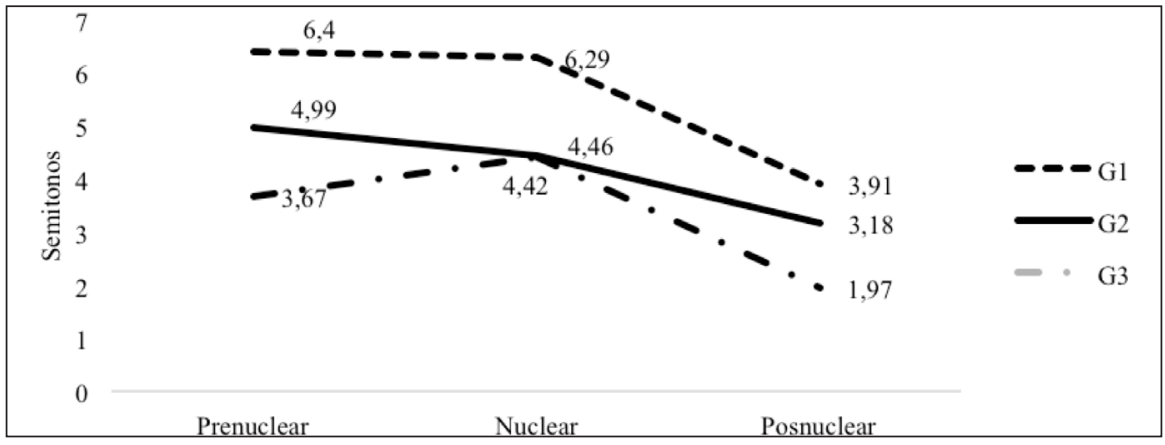

Figura 5. Media de la $f_{0}$ en el tonema por grado de instrucción

\subsection{Diferencias de la $f_{0}$ del tono básico de los informantes con respecto al final del enunciado}

Con relación al movimiento de la $f_{0}$ entre el tono básico y el final del enunciado, se encuentra que la diferencia promedio muestra una flexión de 3.38 st. \pm 4.30 , que refleja un descenso de la sílaba posnuclear. Las medias de las distancias tonales entre el tono básico y la sílaba posnuclear revelan diferencias de altura de la $f_{0}$ de acuerdo con las variables sexo, edad y grado de instrucción (Tabla 4). Sin embargo, el análisis estadístico no revela un efecto significativo de estas variables en la $f_{0}$. Con relación a la variable sexo, los datos muestran que las medias de los descensos tienen valores similares en hombres y mujeres $\mathrm{X}^{2}$ (1) $0.985 \mathrm{p}>0.05$.

\begin{tabular}{ccccc}
\hline \multirow{2}{*}{ Variable } & & \multicolumn{2}{c}{ Tono Básico-Sílaba Posnuclear } & \\
& & Media & Desv.Est & Total \\
\hline \multirow{2}{*}{ Sexo } & Hombres & -3.5 & 3.89 & 45 \\
& Mujeres & -3.07 & 4.71 & 45 \\
\hline \multirow{2}{*}{ Edad } & Gen 1 & -2.49 & 3.08 & 30 \\
& Gen 2 & -4.62 & 5.18 & 30 \\
& Gen 3 & -2.72 & 4.19 & 30 \\
\hline \multirow{2}{*}{ Grado de } & Nivel 1 & -4.62 & 4.79 & 30 \\
Instrucción & Nivel 2 & -2.61 & 3.59 & 30 \\
& Nivel 3 & -2.61 & 4.26 & 30 \\
\hline
\end{tabular}

Tabla 5. Medias de la $f_{0}$ entre las sílabas del tonema por variable grado de instrucción

En cuanto a los grupos generacionales se identifica que los promedios de las diferencias son similares $\mathrm{X}^{2}$ (2) $4.280 \mathrm{p}>0.05$. El valor mayor se encuentra en el grupo de edad 2, entre 
los 35 y 55 años, en comparación con las generaciones 1 y 3. A propósito del nivel de instrucción, los grados 2 y 3 presentaron los mismos valores de diferencia en la $f_{0}$ entre el tono básico y el final del enunciado, sin embargo, el nivel 1 presentó un aumento en la diferencia con relación a los dos restantes; como se mencionó más arriba esta variable no reveló un efecto significativo en la $f_{0}, \mathrm{X}^{2}(2) 2.852 \mathrm{p}>0.05$. Estas diferencias sugieren que los finales de los enunciados declarativos de foco amplio se caracterizan por un declive por debajo del tono medio de los participantes estudiados en un rango de 2.5 a 4.7 st.

\section{CONCLUSIONES}

A partir de la revisión de los enunciados declarativos de foco amplio en el habla limeña, se observa que la configuración entonativa del tonema revela información sociolingüística de los hablantes y, no exclusivamente, información lingüística en cuanto a la identificación de enunciados declarativos en contraste con otro tipo de enunciados. En este sentido, se puede plantear que en datos de habla espontánea el tono de mayor ocurrencia es sostenido en la silaba nuclear $\mathrm{H}^{*}$ con un final sostenido $\mathrm{M} \%$ (entendido como ascensos o descensos menores a $1.5 \mathrm{st}$ ) o descendente L\% (Figura 6). Estos resultados coinciden con los hallazgos de O'Rourke (2015), pero difieren de los resultados obtenidos por García (2015), lo cual puede ser un efecto de tipo de datos analizados dado que en el presente estudio se analizan datos de entrevista ${ }^{5}$.

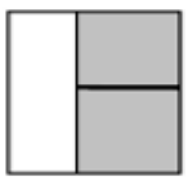

$\mathrm{H}^{*}$

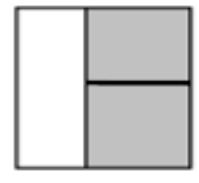

$\mathrm{M} \%$
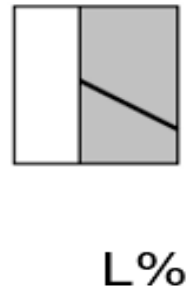

Figura 6. Representación del tonema en enunciados declarativos de foco amplio

Con relación al efecto de las variables sociales en la realización del tonema se encuentra que la altura de la $f_{0}$ se modifica por el efecto de estas variables. El sexo y la edad de los hablantes inciden en la altura alcanzada en la sílaba nuclear. Sin embargo, en la juntura terminal no parece encontrarse un efecto de las variables sociales sexo, generación y grado de instrucción, lo que indica que el final descendente, parece ser común a todos los hablantes y, más estable, en relación con el tipo de enunciado, a diferencia de la sílaba nuclear, aunque es necesario corroborar estos resultados con mayor número de participantes.

Sucede algo similar con la diferencia entre el inicio y el final del enunciado, los hablantes en su mayoría parecen tener una tendencia al declive, que no se ve modificada por el efecto de las variables sociales consideradas dado que no revelan un efecto estadísticamente significado en esta diferencia frecuencial, a reserva de considerar mayor número de participantes.

5 No se debe descartar que la entonación de un enunciado declarativo se modifique en función del tipo de discurso. 
Esta primera aproximación a los datos del corpus PRESEEA permite delinear una metodología para el estudio de datos espontáneos, que en este caso, permitió reconocer que las sílabas que conforman el tonema resultan útiles para la descripción de diferencias fonéticas asociadas a factores sociales, como primer avance para el consecuente análisis de los segmentos pretonemáticos. La importancia de este estudio radica en el reconocimiento de una entonación no marcada como base de comparación para el conocimiento de las variaciones entonativas a nivel discursivo dentro de la variedad limeña y a nivel geoprosódico con otras variedades dialectales.

\section{BIBLIOGRAFÍA}

Ávila, S. (2003). "La entonación del enunciado interrogativo en el español de la ciudad de México", en: Ed. E. Herrera Z. y P. Martín Butragueño. La tonía: dimensiones fonéticas y fonológicas. México: El Colegio de México, pp. 331-355.

Caravedo, R. (coord.)(2008). Corpus sociolingüístico de Lima-PRESEEA. Recuperado de http://preseea.linguas.net/Equipos/Lima.aspx.

Estebas Vilaplana, E. \& Prieto, P. (2008). La notación prosódica del español: una revisión del Sp_ ToBI. Estudios de Fonética Experimental. XVII, p. 263-283.

García, M. (2011). The intonational patters of the peruvian amazonian spanish. MA Thesis. New York: Stonay Brook University.

García, M. (2014). Sobre la duración vocálica y la entonación en el español amazónico peruano. Lengua y Sociedad. Vol. 14, núm. 2, pp. 5-29.

García, M. (2016). The intonation of Peruvian Amazonian Spanish: Rising Accents and Segmental Factors. Ohio: Ohio State University.

Gutiérrez Bravo, R. (2008). La identificación de los tópicos y los focos. Nueva Revista de Filología Hispánica, 56, p. 363-401.

Hidalgo, A. y Padilla, X. (2006). Bases para el análisis de las unidades menores del discurso oral: los subactos. Oralia, 9, p. 109-143.

Hualde, J. I. (2003). "El modelo métrico y autosegmental", en Pilar Prieto (ed.) 2003, Teorías de la entonación. Barcelona: Ariel, pp. 155-184.

Hualde, J.I, y Prieto, P. (2015). "Intonational variation in Spanish: European and American varieties", en S. Frota y P. Prieto (eds). Intonational Variation in Romance. Ed Oxford: Oxford University Press, pp. 350-391.

Martín-Butragueño, P. y Velásquez-Upegui, E. (2014). "Prosodia basada en el uso. Proyecto para el estudio sociolingüístico del español de España y América", presentado en la Reunión del Proyecto PRESEEA en XVII Congresso Internacional da ALFAL, João Pessoa, Paraíba, Brasil, 14-19 de julio.

Navarro Tomás, T. (1948). Manual de pronunciación española. 2da Edición corregida New York: Hispanic Institute.

O'Rourke, E. (2005). Intonation and language contact: A case study of two varieties of Peruvian Spanish. Urbana, Illinois. University of Illinois at Urbana-Champaign. Doctoral dissertation.

Quilis, A. (1993). Tratado de fonología y fonética españolas. Madrid: Gredos.

Pamies Bertrán, A., Fernández Planas, A. M., Martínez Celdrán, E. Ortega Escandell, A. \& Amorós Céspedes, $\mathrm{M}^{\mathrm{a}} \mathrm{C}$. (2002). Umbrales tonales en español peninsular. En:Actas del II Congreso Nacional de Fonética Experimental, pp. 272-278. Sevilla: Universidad de Sevilla.

Pierrehumbert, J. B. (1980). The phonetics and phonology of English intonation. Tesis doctoral. Massachusetts Institute of Technology. Tesis no publicada. Recuperado 15/06/2018,http://faculty. wcas.northwestern.edu/ jbp/publications/Pierrehumbert_PhD.pdf

Pierrehumbert, J. B., \& Beckman, M. E. (1988). Japanese Tone Structure. Cambridge: The MIT Press. 
PRESEEA. Proyecto para el estudio sociolingüístico del español de España y de América. Disponible en http://preseea.linguas.net/.

Prieto, P. (2003). Teorías de la entonación. Ariel: Barcelona.

Prieto, P. y Roseano, P. (eds.) 2010. Transcription of Intonation of the Spanish Language. Münich: Lincom.

Searle, J. (2010). Making the Social World: The Structure of Human Civilization. Oxford: Oxford University Press. [Trad.: Creando el mundo social. La estructura de la civilización humana. Trad. J. Bostelmann].México: Paidós, 2014.

Sosa, J. M. (1999). La entonación del español. Su estructura fónica, variabilidad y dialectología. Madrid: Cátedra.

Velásquez-Upegui, E. y Martín-Butragueño, P. (2018). Guía de Estudios de la prosodia basada en el uso con corpus PRESEEA. Disponible en: http://preseea.linguas.net/Metodolog\%C3\%ADa.aspx

Yule, G. (1996). Pragmatics. Oxford: Oxford University Press. 
